\title{
Reliability Analysis of Safety Intervehicle Communications in a Highway Environment
}

\author{
Yang Wang ${ }^{1}$, Fuqiang $\mathrm{Liu}^{1, *}$, Lijun $\mathrm{Zu}^{2}$ and Nguyen Ngoc $\mathrm{Van}^{3}$ \\ ${ }^{1}$ School of Electronic and Information Engineering, Tongji University, Shanghai, China \\ ${ }^{2}$ China Unionpay, Shanghai, China \\ ${ }^{3}$ School of Electronics and Telecommunications, Hanoi University of Science and Technology, Hanoi, Vietnam \\ ${ }^{*}$ Corresponding author
}

\begin{abstract}
Vehicular network can improve traffic safety by exchange of safety-related messages between the vehicles within the the neighborhood of each other. However, many research related to vehicular network depends heavily on system-level simulation. In this paper, we use tools from stochastic geometry to analyze the reliability of the transmission of periodic message in a highway scenario. A Poission point process (PPP) basedbased network and slotted-aloha MAC scheme is assumed. Three-slope pass loss and rayleigh-lognormal fading are taken into consideration. Coverage probability and packet reception rate (PRR) are adopt as the reliability metrics. We then derive the closed form expressions of coverage probability and PRR. Finally, the accuracy of the analysis is validated via simulation.
\end{abstract}

Keywords-vehicular safety communication; highway; reliability; multi-slope path loss; rayleigh-lognormal fading; PPP

\section{INTRODUCTION}

Vehicular networks can improve traffic safety by exchange of safety-related messages. Periodic message is the most fundamental one defined in vehicular networks, which can help each vehicle keep track with the adjacent vehicles. Generally speaking, the frequency of the periodic message is $10 \mathrm{~Hz}$, and the intended broadcast range is $300 \mathrm{~m}$. Existing standard for intervehicle safety communication is DSRC, which suffers from channel congestion especially in the dense scenario because of the frequent transmission of the periodic message [1].

Many research related to vehicular networks depends heavily on extensive system-level simulation, which is time comsuming. In addition, limited insights can be learned from the simulation results.

Stochastic geometry, especially Poission point process, is widely used in modeling and analysis of wireless networks like ad hoc networks, cellular networks, heterogeneous networks, etc., In the last decades [2]. By averaging over all the spatial patterns, we can get the mathematical expressions of some important performance metrics, from which we can understand how these performance metrics depend on the network parameters and get some useful insights used for protocol optimization and design.

Stochastic geometry has also been applied to intervehicle safety communications. Authors in [3] conclude that the locations of the transmitters in 802.11p tend to be a PPP, and use PPP to approximate the performance in the dense scenario. Works in [3] is extended in [4], where the authors consider both sparse, middle and dense cases, and approximate the coverage probability by a modified matern hard core point process. Reliability of the vehicles near intersections is evaluated in [5]. Multi-lane is considered in both [6] and [7]. In [6], the performance of CSMA based network in a multi-lane highway is investigated. In [7], the authors study the modeling of the multi-hop transmissions in a multi-lane highway setup.

However, both [3-7] consider the simple singular or bounded path loss model and rayleigh fading for mathematical simplicity, which is not the case in the vehicular communication enviroment. Actually, the propagation property is much more complex. From the channel model given by 3GPP in [8], we can know the path loss exponent is distancedependent, and shadowing also exists.

There are some research focusing on the more complicated but more realistic channel models. In [9], the performance of the downlink cellular networks is investigated under multislope path loss model, with a focus on dual-slope model, but the shadowing is not considered. The author in [10] consider nakagami-lognormal fading in the downlink cellular network, but they adopt the simple singular path loss model. The results in both [9] and [10] can not be directly used in vehicular networks, because of the node distribution and performance metrics of the vehicular networks is different from that of the cellular networks.

In this paper, we focus on the transmission of the periodic meaasage in a highway scenario. The more realistic channel model, i.e., three-slope path loss and lognormal shadowing defined in 3GPP [8], are considered. For mathematical tractability, we consider the plain slotted-aloha MAC scheme. Some more involved but efficient MAC schemes is out of the scope of this paper, which we will consider in our future research. Coverage probability and PRR are two performance metrics we are concerned about, of which the mathematical expressions are given in closed-form.

This paper is organized as follows. In section II, system model is introduced. The reliability metrics is analyzed in section III. The accuracy of the analysis results is validated by simulations in section IV. We conclude in section V. 


\section{SYSTEM MODEL}

\section{A. Distributions of the Vehicles}

We consider the highway scenario with a single road, and vehicles are randomly distributed in this road, where we assume the positions of the vehicles follow a 1-D homegeneous PPP $\Phi=\left\{x_{i}\right\}$ with intensity $\lambda$ (we use the term intensity and density interchangeably). With a little abuse of notation, we often represent one vehicle with its location in the rest of the paper. Furthermore, we assume each vehicle generate and transmit a periodic message per broadcast period (i.e., 100ms).

Soltted-Aloha scheme is considered throughout this paper, where each broadcast period is divided into $N$ data slots, and each vehicle choose one data slot randomly in each broadcast period to transimit its periodic message. Each vehicle transmit with a fixed power $P$. We can quickly know from the property of thinning that the locations of the transmitting vehicles and the that of the the receiving vehicles in any data slot form two independent PPP, i.e., $\Phi_{1}$ with intensity $\lambda_{\mathrm{tx}}=\lambda / N$ and $\Phi_{2}$ with intensity $\lambda_{\mathrm{rx}}=(N-1) \lambda / N$ respectively [11]. Each vehicle transmit with a fixed power $P$.

\section{B. Channel Model}

\section{1) Path Loss Model}

We consider the so-called three-slope path loss model which is adopted by 3GPP [8]. Given the length of the wireless link $r$, the path loss function is denoted as

$$
l(r)=\left\{\begin{array}{ccc}
l\left(R_{1}\right) & , \quad 0 \leqslant r<R_{1} \\
A_{1} r^{-\alpha_{1}} & , \quad R_{1} \leqslant r<R_{2} \\
A_{2} r^{-\alpha_{2}} & , \quad r \geqslant R_{2}
\end{array}\right.
$$

Where $R_{1}, R_{2}$ are two critical distances with $R_{1}=3$, $R_{2}=50.35$, and $\alpha_{1}, \alpha_{2}$ are two path loss exponents with $\alpha_{1}=2.27, \alpha_{2}=4$.

Obviously, this function has three slopes in $\mathrm{dB}$ scale, i.e., 0 , $\alpha_{1}, \alpha_{2}$. This function is continuous and bounded in its domain. Indeed, this function is a little bit different from the multi-slope model defined in [9].

\section{2) Shadowing Model}

We assume shadowing follows from a lognormal distribution with mean $\mu$ and standard deviation $\sigma$, and its probability density function (pdf) is given by

$$
f_{1}(x)=\frac{e^{-(\ln y-\mu)^{2} / 2 \sigma^{2}}}{\sqrt{2 \pi} \sigma y}
$$

3) Fading Model

Rayleigh fading is assumed, and its pdf is given by

$$
f_{2}(x)=\rho e^{-\rho x}
$$

where $\rho$ is the mean of the pdf, which we also term as scaling parameter in this paper.

\section{Performance metrics}

If the location of one transmitting vehicle is $x_{0}$, then any vehicle located in $y$ can receive the safety message of $x_{0}$ if and only if

$$
\mathrm{SINR}=\frac{P_{0}}{N_{0}+I}=\frac{P g_{0} l\left(\left|y-x_{0}\right|\right)}{N_{0}+\sum_{x_{i} \in \Phi \backslash x_{0}} P g_{i} l\left(\left|y-x_{i}\right|\right)}>T
$$

where $P_{0}$ is the power received at $\mathrm{y}, I$ is the interference, $T$ is the threshold for successful decoding, $g_{i}$ is the channel gain which equals to the product of shadowing and fading, and $N_{0}$ is the power of the thermal noise. Without loss of any generality, we consider the typical transmitter located in origin, i.e., $x_{0}=o$.

Two performance metrics are considered in this paper. We are first interested in the probability that any vehicle $y$ can successfully receive the periodic message of $x_{0}$, i.e., $\mathbb{P}\left(\operatorname{SINR}^{(y)}>T\right)$, and we call this coverage probability as in [12].

Sometimes it's neccesary to measure the performance of all the vehicles around the transmitter as a whole, because all these vehicles are target receivers. Therefore, we also consider PRR in this paper, which is defined as the pecentage of the nodes that successfully receive a packet from the target transmitter among all the intended receivers [13]. In vehicular network setting, the intended vehicles vehicles in the set $A=\mathcal{B}(0, R)$. Mathematically, PRR can be defined as

$$
p r \triangleq \mathbb{E}_{\Phi}\left[\frac{\sum_{x_{i} \in \Phi(A) \backslash x_{o}} 1\left(\operatorname{SINR}^{\left(x_{i}\right)}>T\right)}{\Phi\left(A \backslash x_{o}\right)} \mid \Phi\left(A \backslash x_{o}\right) \neq 0\right]
$$

where $\mathbf{1}(\cdot)$ is the indicator function. Actually, the concurrent transimitters in $A$ are also intended receivers, but these vehicles can not receive any message because of the constraint of half-duplex.

\section{RELIABILITY ANALYSIS}

The pdf of the channel gain $g$ can be expressed as

$$
f_{g}(x)=\int_{0}^{\infty} e^{-x / \rho y} \frac{1}{\rho y} \frac{e^{-(\ln y-\mu)^{2} / 2 \sigma^{2}}}{\sqrt{2 \pi} \sigma y} d y
$$

The form of the above pdf is complicated at first sight and seems to be intractable. Fortunately, this pdf can be approximated by the sum of several exponential pdf, as described be the following lemma.

Lemma 1: if fading factor follows a rayleigh-lognormal distribution, then its pdf can be approximated with a weighted sum of several exponential distributions 


$$
f_{g}(x) \approx \sum_{n=1}^{N} \omega_{n} f\left(e^{-\left(\sqrt{2} \sigma t_{n}+\mu\right)} / \rho ; x\right)
$$

where $N, t_{n}, \omega_{n}, f(\gamma ; x)$ are the number of the exponential distributions, $n$-th root of the Hermite polynomial, normalized weight given as $2^{N-1} N ! /\left[N^{2} H_{N-1}\left(t_{n}\right)\right]^{2}$, and exponential pdf (i.e., $\left.f(\gamma ; x)=\gamma e^{-\gamma x}\right)$, respectively.

Proof: see [14, Section III]. Note that Rayleigh-lognormal fading is the special case of the nakagami- lognormal fading.

For simplicity, we define $\tau_{n}=\sqrt{2} \sigma t_{n}+\mu$ in the rest of the paper.

\section{A. Coverage Probability}

Lemma 2: The coverage probability of the vehicle located at $y$ can be approximated as

$$
\mathbb{P}\left(\operatorname{SINR}^{(y)}>T\right) \approx \sum_{n=1}^{N} \omega_{n} \exp \left(-\frac{T \tau_{n} N_{0}}{P l(|y|)}\right) \mathcal{L}_{I}\left(\frac{T \tau_{n}}{\operatorname{Pl}(|y|)}\right)
$$

where $\mathcal{L}_{I}(s)=\mathbb{E}_{\Phi_{1}}\left[e^{-s I}\right]$ is the Laplace transform of the interference.

Proof: By definition and lemma 1, we can acquire

$$
\begin{aligned}
& \mathbb{P}\left(\operatorname{SINR}^{(y)}>T\right)=\mathbb{P}\left(h_{0}>\frac{T\left(I+N_{0}\right)}{P l(|y|)}\right) \\
= & \mathbb{E}_{I}\left[\int_{\frac{T\left(I+N_{0}\right)}{P l(|y|)}}^{\infty} f_{h}(x) d x\right] \\
& \stackrel{(a)}{\approx} \sum_{n=1}^{N} \omega_{n} \mathbb{E}_{I}\left[\int_{\frac{T\left(I+N_{0}\right)}{P l(|y|)}}^{\infty} f\left(\tau_{n} ; x\right) d x\right] \\
= & \sum_{n=1}^{N} \omega_{n} \exp \left(-\frac{T \tau_{n} N_{0}}{P l(|y|)}\right) \mathbb{E}_{I}\left[\exp \left(-\frac{T \tau_{n} I}{P l(|y|)}\right)\right] \\
= & \sum_{n=1}^{N} \omega_{n} \exp \left(-\frac{T \tau_{n} N_{0}}{P l(|y|)}\right) \mathcal{L}_{I}\left(\frac{T \tau_{n}}{P l(|y|)}\right)
\end{aligned}
$$

where in step (a), we use the lemma 1.

To obtain the coverage probability, we give the expression of the Laplace transform of the interference in the following lemma.

Lemma 3: The Laplace transform of the interference is given by

$$
\mathcal{L}_{I}(s)=\exp \left(-2 \lambda_{\mathrm{tx}}\left(I_{1}(s)+I_{2}(s)+I_{3}(s)\right)\right)
$$

where

$$
I_{1}(s)=\frac{R_{1}}{1+\left(s P A_{1}\right)^{-1} R_{1}^{2.27}},
$$

$$
v=\left(\frac{1}{s P A_{1}}\right)^{\frac{1}{2.27}}, \theta=\left(\frac{1}{s P A_{2}}\right)^{\frac{1}{4}}
$$

$$
a=\left(v R_{1}\right)^{\frac{1}{4}}, b=\left(v R_{2}\right)^{\frac{1}{4}}, c=\theta R_{2},
$$

$$
\begin{aligned}
I_{2}(s)=\frac{\sqrt{2}}{8 \theta}\left(\ln \frac{c^{2}-\sqrt{2} c+1}{c^{2}+\sqrt{2} c+1}+2 \pi\right. & -2 \arctan (\sqrt{2} c+1) \\
- & 2 \arctan (\sqrt{2} c-1))
\end{aligned}
$$

$$
\begin{aligned}
I_{3}(s)= & \frac{4}{9 v}\left(2 \sum _ { k = 1 } ^ { 4 } \operatorname { s i n } \frac { 4 \pi ( 2 k - 1 ) } { 9 } \left(\arctan \frac{b-\cos \frac{(2 k-1) \pi}{9}}{\sin \frac{(2 k-1) \pi}{9}}\right.\right. \\
& \left.-\arctan \frac{a-\cos \frac{(2 k-1) \pi}{9}}{\sin \frac{(2 k-1) \pi}{9}}\right)-\ln \frac{1+b}{1+a} \\
& \left.-\sum_{k=1}^{4} \cos \frac{4 \pi(2 k-1)}{9} \ln \frac{1-2 b \cos \frac{(2 k-1) \pi}{9}+b^{2}}{1-2 a \cos \frac{(2 k-1) \pi}{9}+a^{2}}\right) .
\end{aligned}
$$

Proof: By definition,

$$
\begin{aligned}
\mathcal{L}_{I}(s) & =\mathbb{E}_{\Phi_{1}, g_{i}}\left[\exp \left(-s \sum_{x_{i} \in \Phi} P_{i} l\left(\left|x_{i}-y\right|\right)\right)\right] \\
& \stackrel{(a)}{=} \mathbb{E}_{\Phi_{1}}\left[\prod_{x_{i} \in \Phi_{1}} \mathbb{E}_{g_{i}}\left[\exp \left(-s P g_{i} l\left(\left|x_{i}-y\right|\right)\right)\right]\right] \\
& \stackrel{(b)}{=} \exp \left(-\lambda_{\mathrm{tx}} \int_{\mathbb{R}}\left(1-\mathbb{E}_{g}[\exp (-s P g l(|x-y|))]\right) d x\right) \\
& =\exp \left(-2 \lambda_{\mathrm{tx}} \int_{0}^{\infty}\left(1-\mathbb{E}_{g}[\exp (-s P g l(|x|))]\right) d x\right) \\
& =\exp \left(-2 \lambda_{\mathrm{tx}} \int_{0}^{\infty}\left(1-\frac{1}{1+s P l(|x|)}\right) d x\right) \\
& =\exp \left(-2 \lambda_{\mathrm{tx}} \int_{0}^{\infty} \frac{1}{1+(s P l(|x|))^{-1}} d x\right) \\
& =\exp \left(-2 \lambda_{\mathrm{tx}}\left(I_{1}(s)+I_{2}(s)+I_{3}(s)\right)\right)
\end{aligned}
$$

where step (a) use the fact that $\left\{g_{i}\right\}$ are i.i.d. random variables, step (b) follows from Probability Generating Functional (PGFL) 
of PPP [12], and

$$
\begin{aligned}
& I_{1}(s)=\int_{0}^{R_{1}} \frac{1}{1+\left(s P A_{1}\right)^{-1} R_{1}^{2.27}} d x=\frac{R_{1}}{1+\left(s P A_{1}\right)^{-1} R_{1}^{2.27}}, \\
& I_{2}(s)=\int_{R_{2}}^{\infty} \frac{1}{1+\left(s P A_{2}\right)^{-1} x^{4}} d x=\frac{1}{\theta} \int_{\mu R_{2}}^{\infty} \frac{1}{1+t^{4}} d t . \\
& I_{3}(s)=\int_{R_{1}}^{R_{2}} \frac{1}{1+\left(s P A_{1}\right)^{-1} x^{2.27}} d x=\frac{1}{v} \int_{v R_{1}}^{v R_{2}} \frac{1}{1+t^{2.27}} d t \\
& \quad(c) \frac{1}{v} \int_{v R_{1}}^{v R_{2}} \frac{1}{1+t^{2.25}} d t \\
& \quad(d) \frac{4}{v} \int_{\left(v R_{1}\right)^{\frac{1}{4}}}^{\left(v R_{2}\right)^{\frac{1}{4}}} \frac{y^{3}}{1+y^{9}} d y,
\end{aligned}
$$

In step (d), we make a variable substitution $y=t^{0.25}$. From [15, eqn. 2.146(2)], we can obtain the expressions of the last two integrations. Note that it's no hard to get the exact expression of $I_{2}(s)$ (one can make a variable substitution $\left.z=t^{0.01}\right)$, but this expression has too many terms. Thus we make a approximation in step (c). We validate the effectiveness of this approximation in section IV.

From lemma 2 and lemma 3, we can derive the expression of the coverage probability.

\section{B. $P R R$}

Lemma 4: For a homogeneous PPP-based network, if the probability that a point located is retained as the transmitter is $p$, then the PRR can be approximated as follows:

$$
p r=\frac{1}{|A|} \int_{A} \mathbb{P}\left(\operatorname{SINR}^{(x)}>T\right) d x
$$

where $|\cdot|$ is Lebesgue measure.

Proof: By the definition of PRR and the property of PPP, we can get

$$
\begin{aligned}
& p r=\mathbb{E}_{\Phi}\left[\frac{\sum_{x_{i} \in \Phi(A) \backslash x_{o}} 1\left(\operatorname{SINR}^{\left(x_{i}\right)}>T\right)}{\Phi\left(A \backslash x_{o}\right)} \mid \Phi\left(A \backslash x_{o}\right) \neq 0\right] \\
& \stackrel{(a)}{\approx} \mathbb{E}_{\Phi_{2}}\left[\frac{\sum_{x_{i} \in \Phi_{2}(A)} 1\left(\operatorname{SINR}^{\left(x_{i}\right)}>T\right)}{\Phi_{2}(A)} \mid \Phi_{2}(A) \neq 0\right] \\
& \stackrel{(b)}{=} \frac{1}{1-\exp \left(-\lambda_{\mathrm{rx}}|A|\right)} \sum_{n=1}^{\infty} e^{-\lambda_{\mathrm{rx}}|A|} \frac{\left(\lambda_{\mathrm{rx}}|A|\right)^{n}}{n !} \frac{1}{|A|^{n}} \\
& \quad \times \iiint_{A^{n}} \frac{\sum_{i=1}^{n} \mathbb{P}\left(\operatorname{SINR}^{\left(x_{i}\right)}>T\right)}{n} d x_{1} d x_{2} \ldots d x_{n} \\
& =\frac{1}{|A|} \int_{A} \mathbb{P}\left(\operatorname{SINR}^{(x)}>T\right) d x
\end{aligned}
$$

where in step (a), we approximate the number of the vehicles in $A$ with the number of the transmitting vehicles in $A$. This approximation is viable because the intensity of $\Phi_{1}$ is much lower than that of $\Phi_{2}$. In step (b), we use the definition of PPP and Bayes formula.

To this end, we can approximate the expression of PRR, as given by the following theorem.

Theorem 1: The PRR can be approximated as

$$
\begin{aligned}
p r \approx & \frac{1}{R} \sum_{n=1}^{N} \omega_{n}\left(R_{1} \exp \left(-a_{n} N_{0}\right) \mathcal{L}_{I}\left(a_{n}\right)\right. \\
& +\left(R_{2}-R_{1}\right) \exp \left(-b_{n} N_{0}\right) \mathcal{L}_{I}\left(b_{n}\right) \\
& +\left(R_{3}-R_{2}\right) \exp \left(-c_{n} N_{0}\right) \mathcal{L}_{I}\left(c_{n}\right) \\
& \left.+\left(R-R_{3}\right) \exp \left(-d_{n} N_{0}\right) \mathcal{L}_{I}\left(d_{n}\right)\right)
\end{aligned}
$$

where $a_{n}=\frac{T \tau_{n} R_{1}^{\alpha_{1}}}{P A_{1}}, b_{n}=\frac{T \tau_{n}\left(R_{2}^{\alpha_{1}+1}-R_{1}^{\alpha_{1}+1}\right)}{P A_{1}\left(1+\alpha_{1}\right)\left(R_{2}-R_{1}\right)}, R_{3}=\frac{R}{2}$,

$$
c_{n}=\frac{T \tau_{n}\left(R_{3}^{\alpha_{2}+1}-R_{2}^{\alpha_{2}+1}\right)}{P A_{1}\left(1+\alpha_{1}\right)\left(R_{3}-R_{2}\right)}, d_{n}=\frac{T \tau_{n}\left(R^{\alpha_{2}+1}-R_{3}^{\alpha_{2}+1}\right)}{P A_{1}\left(1+\alpha_{1}\right)\left(R-R_{3}\right)} .
$$

Proof: One obvious way is to use the result in theorem 1 directly. However, It's difficult to obtain the closed form expressions because of the complexity of (5).

From lemma 2, we can obtain

TABLE I. SIMULATION PARAMETERS

\begin{tabular}{|c|c|}
\hline Parameter & Value \\
\hline Packet Size & 300Byte \\
\hline Bandwidth & $10 \mathrm{M}$ \\
\hline Broadcast Rate & $6 \mathrm{M} \mathrm{bit/s}$ \\
\hline Mean of Shadowing $\mu$ & 0 \\
\hline Standard Deviation $\sigma$ & 3dB \\
\hline Scaling Parameter $\rho$ & $20 \mathrm{dBm}$ \\
\hline Transmit Power $P$ & $-110 \mathrm{dBm}$ \\
\hline SINR Threshold $T$ & $300 \mathrm{dm}$ \\
\hline Intended Tansmission Range $R$ & $10 \mathrm{Km}$ \\
\hline Road Length & $\frac{1}{R} \int_{0}^{R} \mathbb{P}\left(\operatorname{SINR}{ }^{(r)}>T\right) d r$ \\
$\approx$ & $\frac{1}{R} \sum_{n=1}^{N} \omega_{n} \int_{0}^{R} \mathbb{E}_{I}\left[\exp \left(-\frac{T \tau_{n}\left(I+N_{0}\right)}{P l(r)}\right)\right] d r$ \\
$=$ & $\frac{1}{R} \sum_{n=1}^{N} \omega_{n}\left(R_{1} \mathbb{E}_{I}\left[\exp \left(-\frac{T \tau_{n} R_{1}^{\alpha_{1}}\left(I+N_{0}\right)}{P A_{1}}\right)\right]\right.$ \\
$+\int_{R_{1}}^{R_{2}} \mathbb{E}_{I}\left[\exp \left(-\frac{T \tau_{n} r^{\alpha_{1}}\left(I+N_{0}\right)}{P A_{1}}\right)\right] d r$ \\
$\left.+\int_{R_{2}}^{R} \mathbb{E}_{I}\left[\exp \left(-\frac{T \tau_{n} r^{\alpha_{2}}\left(I+N_{0}\right)}{P A_{2}}\right)\right] d r\right)$ \\
\hline
\end{tabular}


To overcome the intractbility, we adopt the following approximation

$$
\frac{1}{b-a} \int_{a}^{b} e^{f(x)} d x \approx \exp \left(\frac{1}{b-a} \int_{a}^{b} f(x) d x\right)
$$

Actually, from Jensen's inequality, we can know that the LHS in (7) is greater than the RHS.

Using (7), we can quickly obtain the result given in the theorem.

\section{NUMERICAL RESULTS}

The parameter used in simulation is given in TABLE I. We can quickly know that the number of avaliable data slots is 250 in each broadcast period from the first three parameters in Table I. The simulation is averaged over $10^{5}$ network realizations for each density. In addition, the number of the exponential pdf $N$ used in lemma 2 is 4 .

In Fig. I, coverage probability is plotted against increasing link length for density $\lambda=0.1$. It can be observed from Fig. I that the analytical results is very close to the simulation results.

Fig. II compared the analytical result and the simulation results of the PRR for different vehicle density. As shown in Fig. II, the difference between the analysis and simulation is very small, which indicate the accuracy of the approximation we used in this paper.

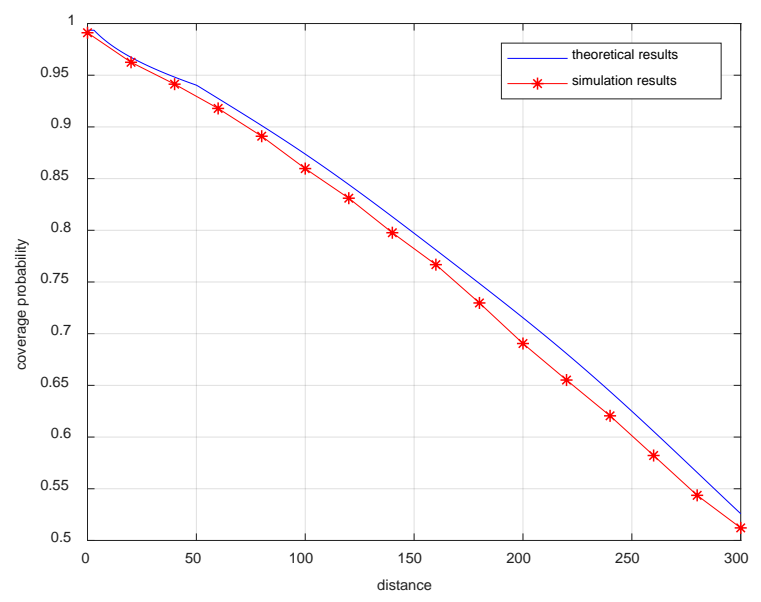

FIGURE I. COVERAGE PROBABILITY FOR DENSITY $\lambda=0.1$

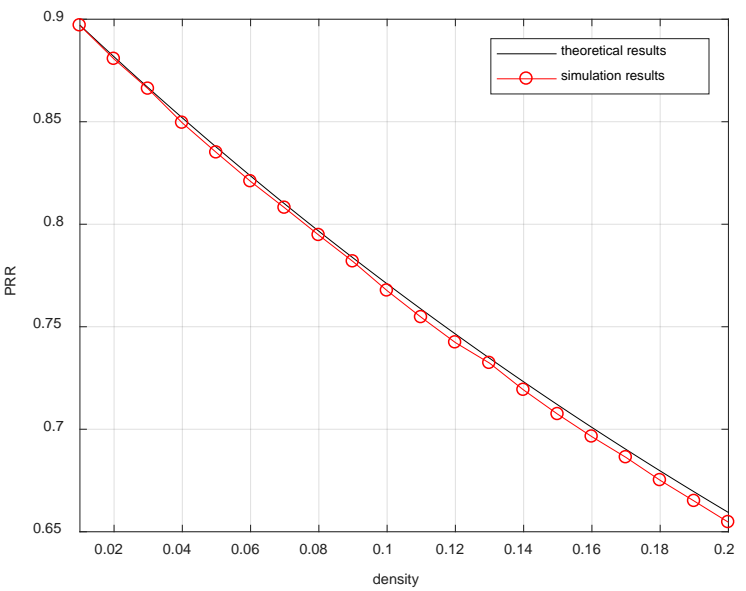

FIGURE II. PRR

\section{CONCLUSION}

In this paper, we use tools from stochastic geometry to analysis two reliability metrics of inter-vehicle safety communication under the highway scenario, with a focus on the transmission of one-hop periodic messages. The locations of the vehicles is modeled as a homogeneous PPP, and slottedaloha is used as the MAC protocol. Three-slope path loss model and rayleigh-lognormal fading model is considered. Through some reasonable approximation, we derive the colsedform expressions of coverage probability and PRR. Finally, we validate the accuracy of the theoretical analysis by simulation.

The work of this paper can be extended in several ways. One possible idea is to consider nakagami-lognormal fading, which may be more complicated but more realistic. Another way is to employ the multi-lane model, which can depict the distribution of the vehicles more accurately. Last but not least, we can focus on the more involved but more efficient MAC protocols for vehicular networks, such as CSMA/CA, TDMA, and so on.

\section{ACKNOWLEDGMENT}

The authors would like to thank the anonymous reviewers for their careful reviews and insightful comments. This work was supported by the National Natural Science Foundation of China (Grant No. 61331009).

\section{REFERENCES}

[1] J. B. Kenney, "Dedicated short-range communications DSRC standards in the united states," Proc. IEEE, vol. 99, no. 7, pp. 1162-1182, 2011.

[2] M. Haenggi, J. G. Andrews, F. Baccelli, O. Dousse, and M. Franceschetti, "Stochastic geometry and random graphs for the analysis and design of wireless networks,"IEEE J. Sel. Areas Commun., vol. 27, no. 7, pp. 1029-1046, Sep. 2009.

[3] T. V. Nguyen, F. Baccelli, K. Zhu, S. Subramanian, and X. Wu, "A performance analysis of CSMA based broadcast protocol in VANETs," in Proc. IEEE INFOCOM, Turin, Italy, Apr. 2013, pp. 2805-2813.

[4] Z. Tong, H. Lu, M. Haenggi, and C. Poellabauer, “A stochastic geometry approach to the modeling of DSRC for vehicular safety 
communication,” IEEE Trans. Intell. Transp. Syst., vol. 17, no. 5, pp. 1448-1458, May 2016.

[5] E. Steinmetz, M. Wildemeersch, T. Q. S. Quek, and H. Wymeersch, "A stochastic geometry model for vehicular communication near intersections," in 2015 IEEE Globecom Workshops (GC Wkshps), Dec. 2015, pp. 1-6.

[6] M. J. Farooq, H. ElSawy, and M. S. Alouini, "Modeling inter-vehicle communication in multi-lane highways: A stochastic geometry approach,” in Proc. IEEE VTC, Boston, MA, USA, Sep. 2015, pp. 1-5.

[7] M. J. Farooq, H. ElSawy, and M. S. Alouini, "A stochastic geometry model for multi-hop highway vehicular communication,” IEEE Trans. Wireless Commun., vol. 15, no. 3, pp. 2276-2291, Mar. 2016.

[8] 3GPP, "Study on LTE-based V2X services (release 14),"3rd Generation Partnership Project (3GPP), Sophia Antipolis Valbonne, France, Tech. Rep. 36.885 V14.0.0, Jun. 2016.

[9] X. Zhang and J. G. Andrews, "Downlink cellular network analysis with multi-slope path loss models," IEEE Trans. Commun., vol. 63, no. 5, pp. 1881-1894, May 2015.

[10] K. Cho, J. Lee, and C. G. Kang, "Stochastic geometry-based coverage and rate analysis under nakagami and log-normal composite fading channel for downlink cellular networks,” IEEE Commun. Lett., vol. 21, no. 6, pp. 1437-1440, Jun. 2017.

[11] F. Baccelli and B. Blaszczyszyn, Stochastic geometry and wireless networks, vol. 1. Norwell, MA, USA: Now Publishers, 2009.

[12] X. Lin, R. Ratasuk, A. Ghosh, and J. G. Andrews, "Modeling, analysis, and optimization of multicast device-to-device transmissions," IEEE Trans. Wireless Commun., vol. 13, no. 8, pp. 4346-4359, Aug. 2014.

[13] X. Ma, J. Zhang, and T. Wu, "Reliability analysis of one-hop safetycritical broadcast services in VANETs,” IEEE Trans. Veh. Technol., vol. 60, no. 8, pp. 3933-3946, Oct. 2011.

[14] S. Atapattu, C. Tellambura, and H. Jiang, "A mixture gamma distribution to model the snr of wireless channels," IEEE Trans. Wireless Commun., vol. 10, no. 12, pp. 4193-4203, Dec. 2011.

[15] I. S. Gradshteyn and I. M. Ryzhik, Table of Integrals, Series, and Products, 7th ed. New York, NY, USA: Academic, 2007. 